\title{
Reversal of Hartmann's procedure through the stomal side: a new even more minimal invasive technique
}

\author{
Jefrey Vermeulen · Wietske Vrijland • \\ Guido H. H. Mannaerts
}

Received: 29 December 2007/ Accepted: 26 May 2008/Published online: 12 July 2008

(C) The Author(s) 2008

\begin{abstract}
Background Several minimal invasive, mainly laparoscopic-assisted, techniques for reversal of Hartmann's procedure (HP) have been published. The purpose of this pilot study was to assess a minimal invasive procedure through the stomal site that may compare favorably with open or laparoscopic-assisted procedures in terms of operative time, hospital stay and postoperative complications.

Methods HP reversal through the stomal side was attempted in 13 consecutive patients. Lysis of intraabdominal adhesions was done manually through an incision at the formal stoma side, without direct vision between thumb and index finger. The rectal stump was identified intra-abdominally using a transanal rigid club. A manually controlled stapled end-to-end colorectal anastomosis was created.

Results Mean duration of operation was 81 min (range 58-109 min); mean hospital stay was 4.2 days (range 27 days). In two patients the procedure was converted because of strong adhesions in the lower pelvic cavity around the rectal stump that could not be lysed manually safely. No complications occurred in the patients in whom reversal was completely done through the stomal site.

Conclusions In our opinion, restoration of intestinal continuity through the stomal side after HP is a feasible operation, without need for additional incisions. In the
\end{abstract}

\footnotetext{
J. Vermeulen

Department of Surgery, Erasmus University Medical Center, Dr. Molenwaterplein 40, 3015 GD Rotterdam, The Netherlands e-mail: j.vermeulen.1@erasmusmc.nl

W. Vrijland · G. H. H. Mannaerts ( $)$

Deptarment of Surgery, St. Franciscus Gasthuis, Kleiweg 500, 3045 PM Rotterdam, The Netherlands

e-mail: g.mannaerts@SFG.nl
}

hands of a specialist gastrointestinal surgeon this technique can be attempted in all patients, as conversion to a laparoscopic-assisted or an open procedure can be performed when necessary.

Keywords Hartmann's procedure - Stoma - Reversal · Minimal invasive

Restoration of bowel continuity after Hartmann's procedure (HP) is a technically challenging operation, associated with significant morbidity, with reported anastomotic leak rates of $4-16 \%$ and a mortality of up to $4 \%$ [1]. These rates can be as high as $30 \%$ and $14 \%$, respectively, after stoma reversal in patients who had undergone a HP for complicated diverticulitis [2,3]. This is the main reason why approximately $40 \%$ of the patients after HP will never undergo restoration of digestive continuity [1]. Besides risk factors such as the patients characteristic's $[4,5]$ and disease etiology $[1,2]$ that cannot be altered, the most important risk factor for morbidity is the technique used for restoration of bowel continuity [6-8].

Although many technical variations are described for reversal of HP, the optimal technique is still a matter of debate. Minimal invasive techniques (laparoscopic or endoscopic assisted), although lasting longer and technically challenging, seem to have advantages regarding less postoperative pain and disability, shorter hospital stay, and better cosmetics [8-11]. However, also in laparoscopic HP reversal all adhesions in the midline and pelvis need to be loosened. This may increase morbidity, i.e., postoperative paralytic ileus and small bowel lacerations.

This study describes a new even less invasive technique for reversal of HP through the stomal site, without the need 
for laparoscopic (or endoscopic) assistance. The feasibility of this technique and its outcome in terms of operative time and morbidity was assessed in a consecutive series of 13 patients.

\section{Materials and methods}

Since August 2005 a total of 13 consecutive patients underwent reversal of a Hartmann's procedure through the stomal side at the Sint Franciscus Gasthuis Rotterdam, The Netherlands. All procedures were performed by one consultant surgeon (G.H.H.M.). Patients' data and results were recorded prospectively. The only exclusion criterion for trans-stomal restoration of bowel continuity was an accompanying abdominal wall hernia that needed correction with a mesh.

\section{Surgical technique}

Preoperative bowel preparation consists out of an anal clysma. Broad-spectrum antibiotic prophylaxes $(1000 \mathrm{mg}$ Kefzol $^{\circledR} / 500 \mathrm{mg}$ Flagyl ${ }^{\circledR}$ ) are administered before surgery. Patients are placed in the lithotomy position and the first surgeon stands at the left of the patient throughout the operation.

First the colostomy is closed with a running suture in order to continue with a clean operation.

Then the patient is scrubbed with Povidon ${ }^{\circledR}$ and the surgeon switches gloves. The stoma is released, taking a small amount of surrounding skin with it. The length of the incision at the stomal side must be just large enough for the surgeon to put his right hand intra-abdominally. The descending colon stump is brought outside the abdomen and adhesions to the left colon are carefully loosened by sharp dissection as long as it is visible. Further loosening of adhesions of the left colon is performed manually with index finger or between thumb and index finger in order to create enough length of the descending colon to reach the pelvic cavity. To achieve this, mobilization of the splenic flexure is seldom necessary, even if this has not been performed at primary surgery. If enough bowel length is created, clamps are placed at the end. The very distal part of the bowel with its attached skin is removed. An anvil of a circular stapler $(31 \mathrm{~mm})$ is placed intraluminal. The stump is closed using a linear stapler. The tip of the stapler anvil is brought through the colon wall just near the staple line and tied by a purse-string suture. This bowel segment including anvil is brought intra-abdominally.

Next the surgeon's right hand is placed intra-abdominally through the former stoma defect. Adhesions in the pathway to the distal (rectosigmoid) stump are gently loosened in a careful digital blind fashion. The left hand is used to introduce a rigid club transanal to identify the rectal

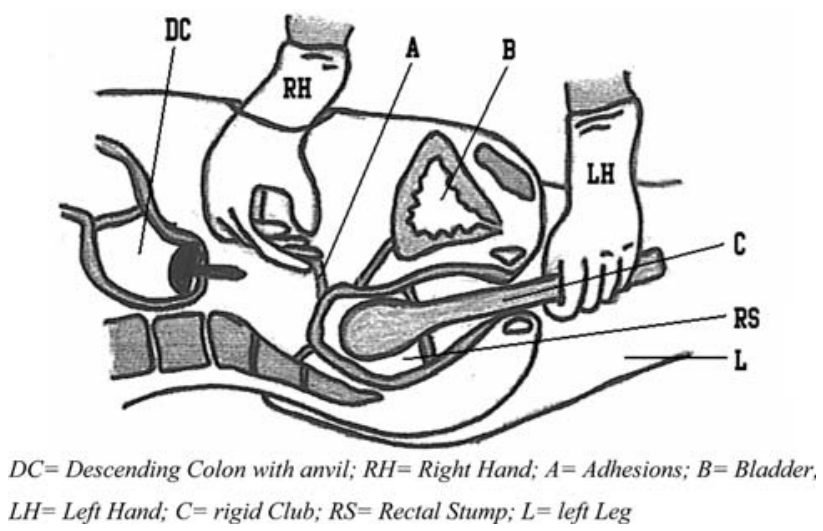

Fig. 1 Manual lysis of adhesions at the tip of the rectal stump, which was identified using a rigid club. Previously the anvil of a circular stapler was placed intraluminal of the descending colon. DC, descending colon with anvil; $\mathrm{RH}$, right hand; A, adhesions; $\mathrm{B}$, bladder; LH, left hand; C, rigid club; RS, rectal stump; L, left leg

stump. The rectal stump is manually gently lysed from small bowel adhesions by the surgeon's right hand (Fig. 1).

Consecutively the circular stapler is introduced into the rectal stump. The pin of the circular stapler is passed through the rectal wall and then removed under digital control. Then the anvil is attached to the tip of the circular stapler. Before firing the circular stapler the proximal bowel segment is manually checked for rotation and interposition of small bowel, abdominal fat or the vagina wall. After firing the stapler, the integrity of the doughnuts of the functional end-to-end anastomosis is inspected. The stoma opening is closed with resorbable sutures (Vicryl 3.0) as well as the skin (Monocryl 3.0).

In case of firm adhesions that cannot be lysed manually the operation is converted to a laparoscopic-assisted procedure. However, when the adhesions are very firm, direct conversion to an open (laparotomy) procedure is executed.

\section{Results}

Patient characteristics are listed in Table 1. Of the 13 patients that were assigned for reversal of Hartmann's procedure through the stomal site, two needed direct conversion to an open procedure (laparotomy) due to very firm adhesions in the pelvis that were not even tried to be loosened manually ( $15 \%$ conversion rate). One patient who underwent direct conversion to an open procedure because of firm adhesions not suitable for manual lysis developed an anastomotic leakage.

In one patient the anastomosis was inspected using a $30^{\circ}$ telescope via the incision at the stomal side. No additional incisions were necessary in the 11 patients in whom reversal was accomplished through the stomal side. They all were without postoperative complications and could leave the hospital within 1 week (Table 2). 
Table 1 Patient demographics $(n=13)$

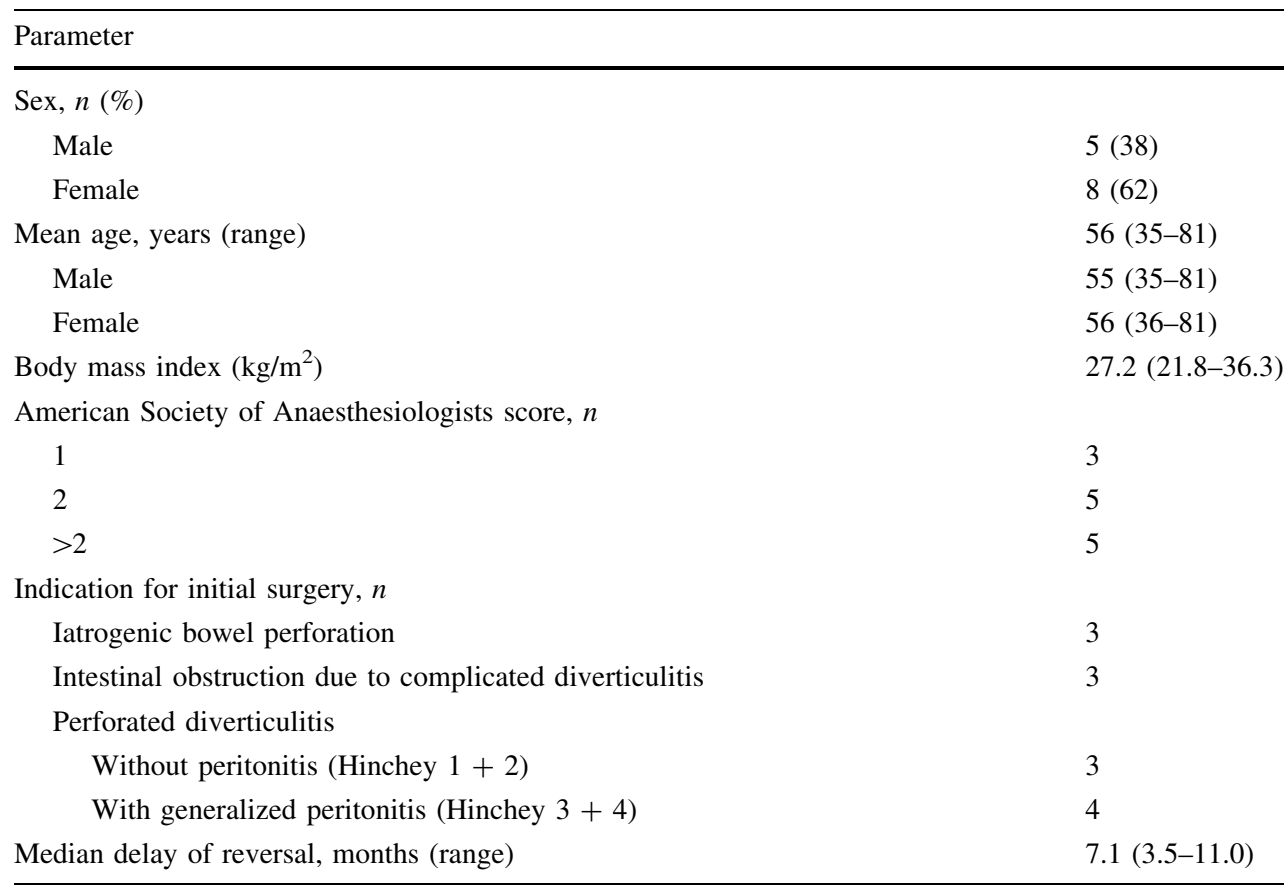

Table 2 Results after reversal of Hartmann's procedure through the stomal side

\begin{tabular}{lllll}
\hline $\begin{array}{l}\text { Number of transstomal completed } \\
\text { patients }\end{array}$ & $\begin{array}{l}\text { Mean operation time } \\
(\mathrm{min})\end{array}$ & $\begin{array}{l}\text { Mean postoperative hospital stay } \\
\text { (days) }\end{array}$ & $\begin{array}{l}\text { Length of incision } \\
(\mathrm{cm})\end{array}$ & $\begin{array}{l}\text { Number of anastomotic } \\
\text { leaks }\end{array}$ \\
\hline$(n=11)$ & $81(58-109)$ & $4.2(2-7)$ & $9.2(7.5-11)$ & 0 \\
\hline
\end{tabular}

In long-term follow-up one patient developed an incisional hernia at the stomal site 12 months postoperatively, which did not need surgical correction. Overall the patients showed quick recovery and were very content with the aesthetic outcome.

\section{Discussion}

The standard surgical approach to the restoration of continuity has been by laparotomy. Minimally invasive surgery has gained popularity, because of less postoperative pain and disability, shorter postoperative hospital stay, better cosmetics, and a faster return to work [9-11]. By significantly reducing the operative trauma, reports have shown decreased postoperative recovery time and surgically related stress [8].

Our even more minimal invasive blind manual transstomal technique is a feasible technique and supposed to have several advantages, such as shorter operation time, less need for adhesiolysis, and faster recovery, compared with open and even with laparoscopic-assisted procedures. Moreover, HP reversal through the stomal side has the advantage over laparoscopic-assisted HP reversal that no additional incisions has to be made to place the trocars, which is supposed to improve aesthetics.
Open Access This article is distributed under the terms of the Creative Commons Attribution Noncommercial License which permits any noncommercial use, distribution, and reproduction in any medium, provided the original author(s) and source are credited.

\section{References}

1. Banerjee S, Leather AJM, Rennie JA, Samano N, Gonzalez JG, Papagrigoriadis S (2005) Feasibility and morbidity of reversal of Hartmann's. Colorectal Dis 7:454-459

2. Salem L, Flum DR (2004) Primary anastomosis or Hartmann's procedure for patients with diverticular peritonitis? A systematic review. Dis Colon Rectum 47:1953-1964

3. Maggard MA, Zingmond D, O'Connell JB, Ko CY (2004) What proportion of patients with an ostomy (for diverticulitis) get reversed? Am Surg 70:928-931

4. Roque-Castellano C, Marchena-Gomez J, Hemmersbach-Miller M, Acosta-Merida A, Rodriguez-Mendez A, Farina-Castro R, Hernandez-Romero J (2007) Analysis of the factors related to the decision of restoring intestinal continuity after Hartmann's procedure. Int J Colorectal Dis 22:1091-1096

5. Salem L, Anaya DA, Roberts KE, Flum DR (2005) Hartmann's colectomy and reversal in diverticulitis: a population-level assessment. Dis Colon Rectum 48:988-995

6. Pittman DM, Smith LE (1985) Complications of colostomy closure. Dis Colon Rectum 28:836-843

7. Detry RJ, Kartheuser A, Delriviere L, Saba J, Kestens PJ (1995) Use of the circular stapler in 1000 consecutive colorectal anastomoses: experience of one surgical team. Surgery 117:140-145 
8. Holland JC, Winter DC, Richardson D (2002) Laparoscopically assisted reversal of Hartmann's procedure revisited. Surg Laparosc Endosc Percutan Tech 12:291-294

9. Sosa JL, Sleeman D, Puente I, McKenney MG, Hartmann R (1994) Laparoscopic-assisted colostomy closure after Hartmann's procedure. Dis Colon Rectum 37:149-152
10. Navarra G, Occhionorelli S, Marcello D, Bresadola V, Santini M, Rubbini M (1995) Gasless video-assisted reversal of Hartmann's procedure. Surg Endosc 9:687-689

11. Rosen MJ, Cobb WS, Kercher KW, Heniford BT (2006) Laparoscopic versus open colostomy reversal: a comparative analysis. J Gastrointest Surg 10:895-900 